\title{
EL CRECIMIENTO URBANO DE LEBRIJA (SEVILLA) EN EL SIGLO XVI.
}

\section{URBAN GROWTH OF LEBRIJA (SEVILLE, SPAIN) IN THE $16^{\text {TH }}$ CENTURY.}

\author{
Juan Antonio Caro Cals \\ juancarocals@arquired.es
}

Recibido: febrero, 2012.

Versión final aceptada: noviembre, 2012.

PALABRAS CLAVE: Edad Moderna, expansión extramuros, tejido urbano, Barrionuevo. KEYWORDS: Modern Age, sprawl outside the walls, urban fabric, Barrionuevo.

\section{RESUMEN}

Se expone en el presente trabajo un estudio de la expansión urbana de Lebrija (Sevilla) centrado en el siglo XVI, momento que asiste a la ocupación del terreno extramuros de la villa, ceñida hasta entonces por la cerca almohade. Asimismo, se incluye una restitución gráfica aproximada de la red viaria de la época, su estructura urbana y su parcelario.

\section{ABSTRACT}

The aim of this paper is to expose the urban expansion of Lebrija (Seville, Spain) focusing on the $16^{\text {th }}$ century, when the city exceeds the islamic walls to occupy the land outside. A planimetry of the road network and urban pattern at the time is included. 


\section{OBJETO DEL ESTUDIO Y METODOLOGÍA.}

Con el inicio de la Edad Moderna las principales poblaciones de Andalucía Occidental reciben un impulso urbanístico liberador que las lleva a rebasar sus recintos murados. Jerez de la Frontera abandona el perímetro de su medina a finales del siglo XV y principios del XVI (Amaya, R. L., 2010, 417). Sevilla experimenta en el Quinientos una «eclosión demográfica» que, secundada por un fuerte desarrollo edilicio, ve nacer los arrabales de La Carretería, La Cestería, Los Humeros, etc (Del Pozo, A., 1996, 39). Los núcleos urbanos del Bajo Guadalquivir no son ajenos a este fenómeno motivado por el alejamiento definitivo de la frontera con los territorios bajo dominación musulmana y la inestabilidad que los conflictos bélicos de la zona aparejaban (figura 1).

Figura 1. Situación de la localidad.

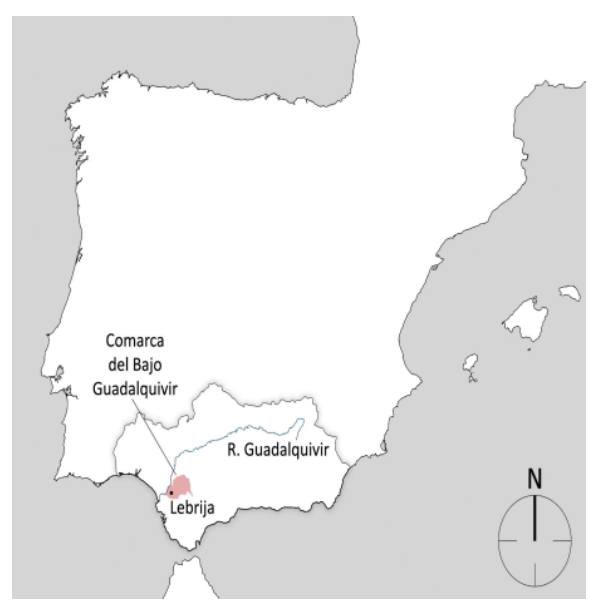

En el caso de Lebrija, el siglo XVI brindará a la villa un primer momento de auge constructivo, una expansión suburbana que más tarde consolidan los siglos XVII y XVIII. La cerca, despojada de su función defensiva — al igual que el castillo-, es amortizada por nuevas viviendas, y posiblemente objeto de la apertura de nuevos postigos. La ciudad crea en esta época un tejido urbano de trazado regular, ejes radiales y plazas abiertas cuya formación, planificada o espontánea, pervive y llega hasta nuestros días prácticamente en su estado original (Pérez, M., 2005).

Un dato a tener en cuenta es que la extensión de este crecimiento moderno ocupa la casi totalidad del área que actualmente delimitan tanto el Conjunto Histórico Artístico como el Yacimiento Arqueológico Urbano de la villa ${ }^{1}$ (figura 2); Lebrija no volvió a experimentar en siglos posteriores y hasta cronologías contemporáneas un desarrollo urbano de semejante magnitud.

\footnotetext{
${ }^{1}$ Área que coincide en gran medida con la representada, en el siglo XIX, en el "Plano Pintoresco de la Villa de Lebrija" de José López Alegría.
} 
Figura 2. Planimetría de la población.

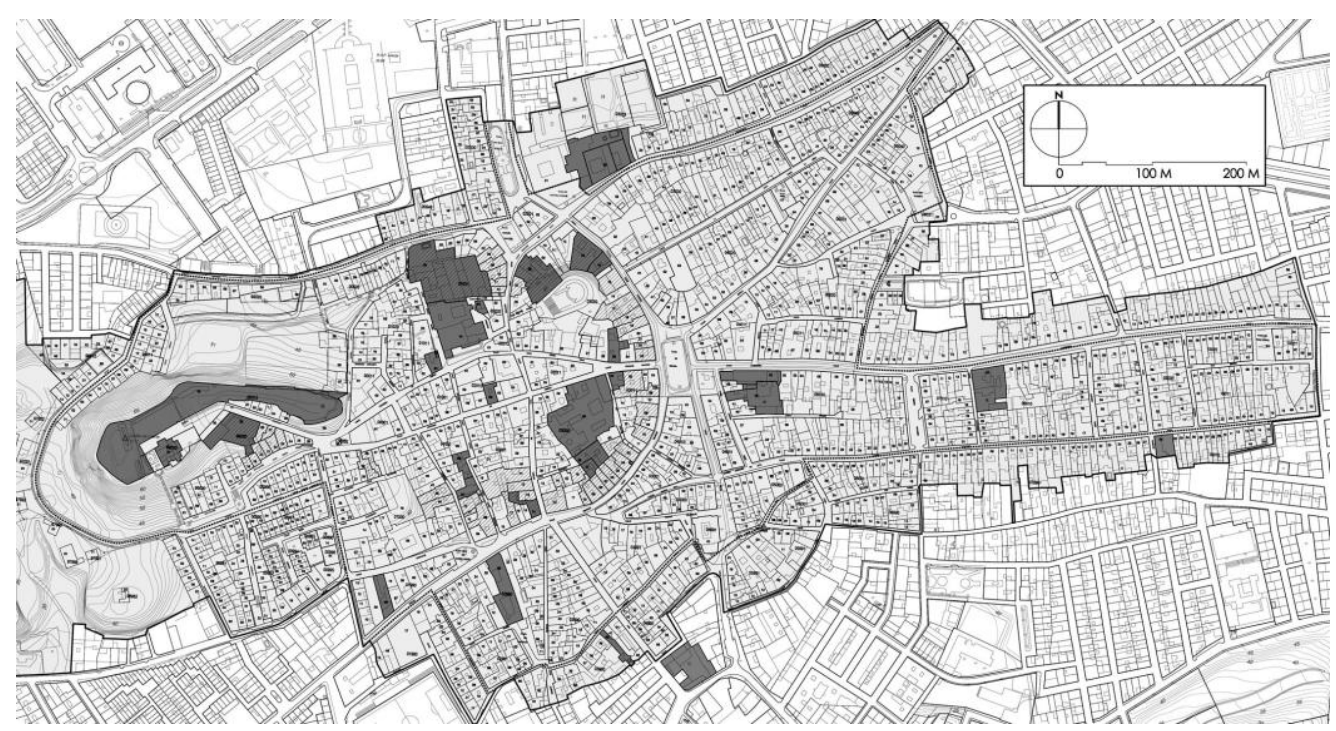

Se sombrean los ámbitos del Conjunto Histórico y del Yacimiento Arqueológico Urbano. Detalle extraído del plano PC-5, Planos de Protección y Catálogo, del Plan General de Ordenación Urbanística de Lebrija.

A la vista de los estudios desarrollados por diversos autores sobre la evolución urbana de Lebrija, también expuesta en el Plan General de Ordenación Urbana de la ciudad, se pretende con este trabajo lanzar una hipótesis de restitución -centrada en el siglo $\mathrm{XVI}$ - de la estructura urbana de la Lebrija extramuros y su desarrollo a lo largo de dicho siglo (algo ya examinado en Tomassetti, J. M., 1997, 262). Se trata de un ejercicio de análisis basado en los mecanismos teóricos de crecimiento de las ciudades, análisis sostenido por el registro documental y validado - siempre que se dispongan de intervenciones para ello- por la arqueología. Así, se han tenido en cuenta para este estudio los siguientes parámetros:

- La topografía y configuración del espacio físico donde asienta la población,

- La antigua red hidrológica,

- Los flujos de escala territorial y local,

- Las cronologías aportadas por las intervenciones arqueológicos llevadas a cabo en el núcleo urbano,

- Los datos recogidos en la documentación y la planimetría históricas,

- Las pervivencias formales, vivas o fósiles, extraíbles del análisis del catastro actual (alineaciones, medianeras, viario, líneas de crujía, líneas de parcelario...),

- El estudio del nomenclátor antiguo. 


\section{ASENTAMIENTO Y RED DE CAMINOS.}

El asentamiento de la villa de Lebrija presenta una situación geográfica en cul-de-sac. Recoge, al menos, dos flujos territoriales procedentes del nordeste: uno que bordea el antiguo límite ribereño del paleoestuario del Guadalquivir hasta Sevilla, y otro que se aleja del entorno palustre y penetra hacia el interior alcanzando poblaciones como Espera o Utrera. Estos dos caminos, junto a un tercero que se aleja en dirección sureste hacia Arcos de la Frontera, debieron ser los flujos originales de la red de comunicación del asentamiento, flujos que penetran en la villa a través de las calles Sevilla, Corredera y Arcos respectivamente, luego convergen en la plaza de España - antesala de la antigua puerta de Sevilla de la cerca urbana- y cruzan el recinto de la madina almohade para subir el Cerro del Castillo y dar fin a su recorrido (figura 3).

Figura 3. Recorridos originales del asentamiento.

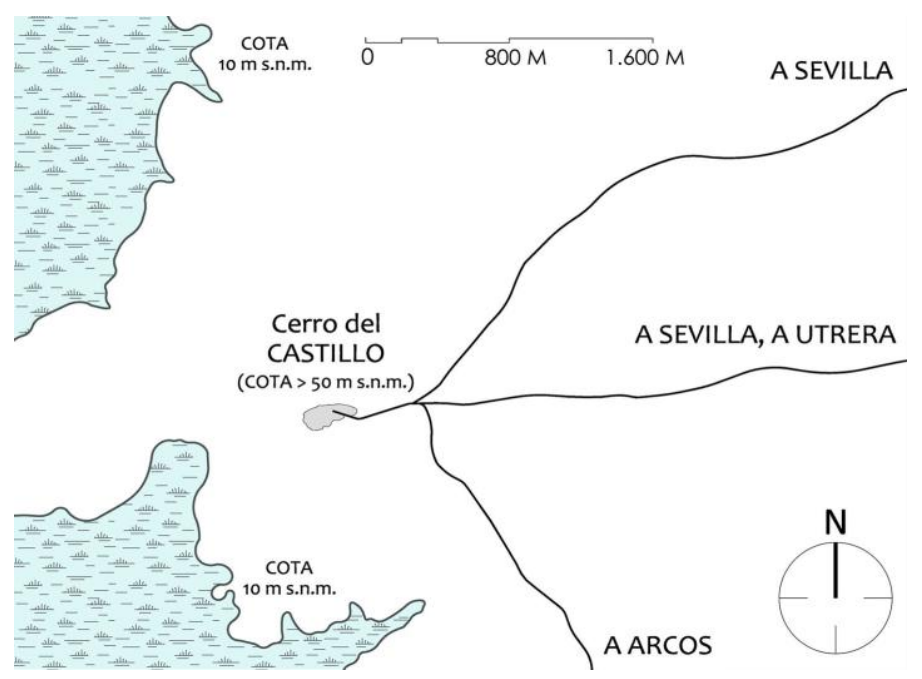

La cercanía del cerro al paleoestuario del Bajo Guadalquivir justifica la ausencia de vías de comunicación terrestres por el arco noroeste del asentamiento, ocupado por esteros y lagunas sujetos al régimen mareal oceánico. Esta situación va a cambiar a lo largo de la Edad Media. La paulatina colmatación del antiguo estuario permite la aparición de dos vías cuyos recorridos atraviesan terrenos hasta ahora impracticables: las veredas de Sanlúcar de Barrameda y Jerez de la Frontera; si bien estos caminos pueden haber estado en uso en períodos anteriores (asumiendo una alternancia de recorridos terrestres con trayectos fluviales), es en este momento, y motivado por el alejamiento de la línea lacustre - que apareja o aumenta su carácter transitable-, cuando ambos caminos adquieren un mayor uso e importancia, adquiriendo igualmente su carácter de condicionantes en la posterior expansión urbana.

Un esquema de geometría radial completa estos cinco recorridos matrices (figura 4). Se trata de una red viaria que conecta el núcleo urbano con el entorno agrícola de la villa y con poblaciones menores alejadas de las rutas principales. Esta red debía estar 
ya en uso a la llegada de los castellanos a mediados del siglo XIII. La Memoria de Información del Plan General de Ordenación Urbanística de Lebrija cataloga y describe pormenorizadamente estos caminos rurales y vías pecuarias que bajo las denominaciones de "vereda», "cañada», "colada» o "cordel», configuran el sistema de comunicaciones del hinterland de la villa.

Figura 4.

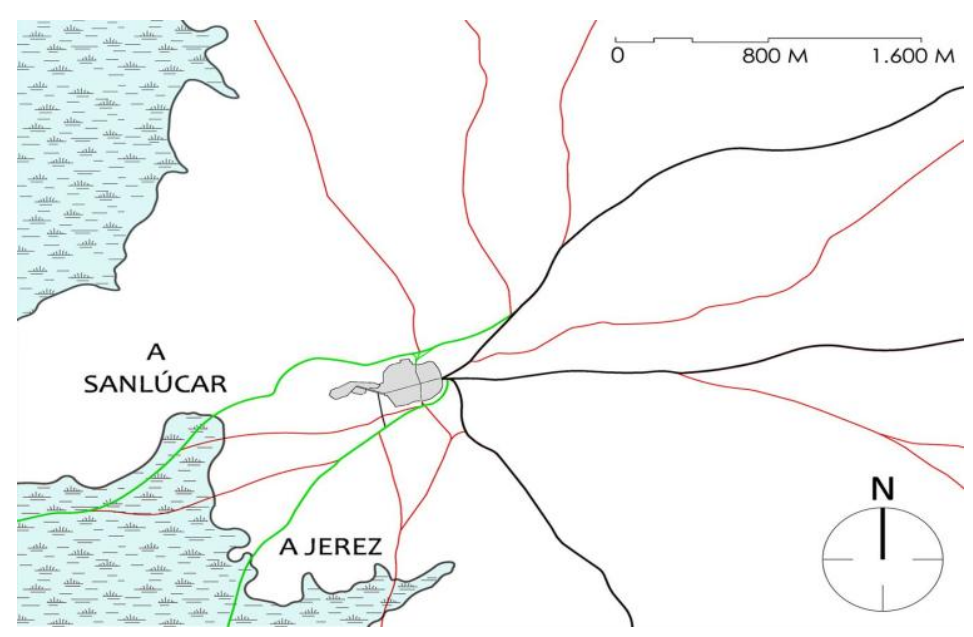

En la edad Media se añaden las veredas con dirección a Sanlúcar y a Jerez de la Frontera (en verde). Caminos secundarios completan la red viaria, de esquema radial (en rojo).

\section{LEBRIJA A FINALES DE LA EDAD MEDIA.}

Los registros documentales que permiten extraer datos sobre la articulación urbana de Lebrija aparecen en el umbral del siglo XV. La fecha estimada para el comienzo de su expansión urbana más allá de los límites de su cerca defensiva nos llevan a las postrimerías de dicho siglo, con la conquista del reino de Granada y la llegada al Nuevo Mundo como hitos cronológicos de partida. La ciudad aún no ha desbordado sus murallas, pero ya suponemos la existencia del cinturón de edificaciones que parasita ambas caras de la misma apoyando sus traseras sobre la cerca. Todavía en el siglo XV subsisten espacios libres sin edificar en el área intramuros, como la manzana comprendida entre la calle Naranjos y la calle José Sánchez de Alva (Bellido, J., 1985: 458).

Fuera de las murallas sólo tenemos noticias de una posible implantación edilicia a lo largo la calle Cataño (Bellido, 1985: 441). Su cercanía al núcleo amurallado y su conexión entre la plaza de España y la fuente abrevadero ubicada por entonces en la actual plaza Juan Díaz de Solís hacían este recorrido apto para una ocupación temprana; lo cual no descarta la presencia de instalaciones de uso agropecuario, industrial o residencial en otras vías. Candidatas a este primer ejercicio de expansión urbana más allá de la ciudad almohade son las calles Corredera y Sevilla. Su condición de flujos territoriales, comentada en el apartado anterior, las hacen susceptibles de acoger inmuebles puntuales, ya sean viviendas, huertas, establos o cortinales. Bellido avala esta conjetura en el caso de la calle Corredera al referir la antigüedad del topónimo - «Corredera del Arco»- en el callejero del siglo XV (Bellido, J., 1985, 444). 
También aporta la referencia de una huerta, la de Gómez Benítez de Medina (Bellido, J., 1985, 470), que ocupaba en este siglo el sector comprendido entre las calles Sevilla, Corredera, Molinos y Eduardo Dato hasta las Ilamadas "Caleras de Pedro García» (el entorno de la actual plaza del Pajarete). Las márgenes de esta gran parcela rústica debieron ser ocupadas progresivamente desde la plaza de España hacia el exterior de la villa, si bien no hay constancia de expedientes constructivos en la zona para este momento.

Otra vía extramuros cuyo nombre medieval se conoce es el de la calle Andrés Sánchez de Alva, continuación de Benito Vela. Se trata de una vía secundaria que daba comienzo en la Vereda Real de Jerez a la altura de la puerta de Rota (junto a la Capilla de la Veracruz), cruzaba el arroyo Zangalabota y corría paralela a la calle Corredera para unirse finalmente a ella más allá del límite del casco histórico tradicional. Asociada al proceso de expansión urbana de los siglos XVIII y XIX pudo, no obstante, ser terreno para la formación espontánea de tejido urbano no consolidado en el siglo XV. Documentos de la época la nombran como «calle de las Fontanillas». Estaba dividida en cinco tramos (Bellido, J., 1985, 435).

Figura 5. La villa a finales del siglo XV.

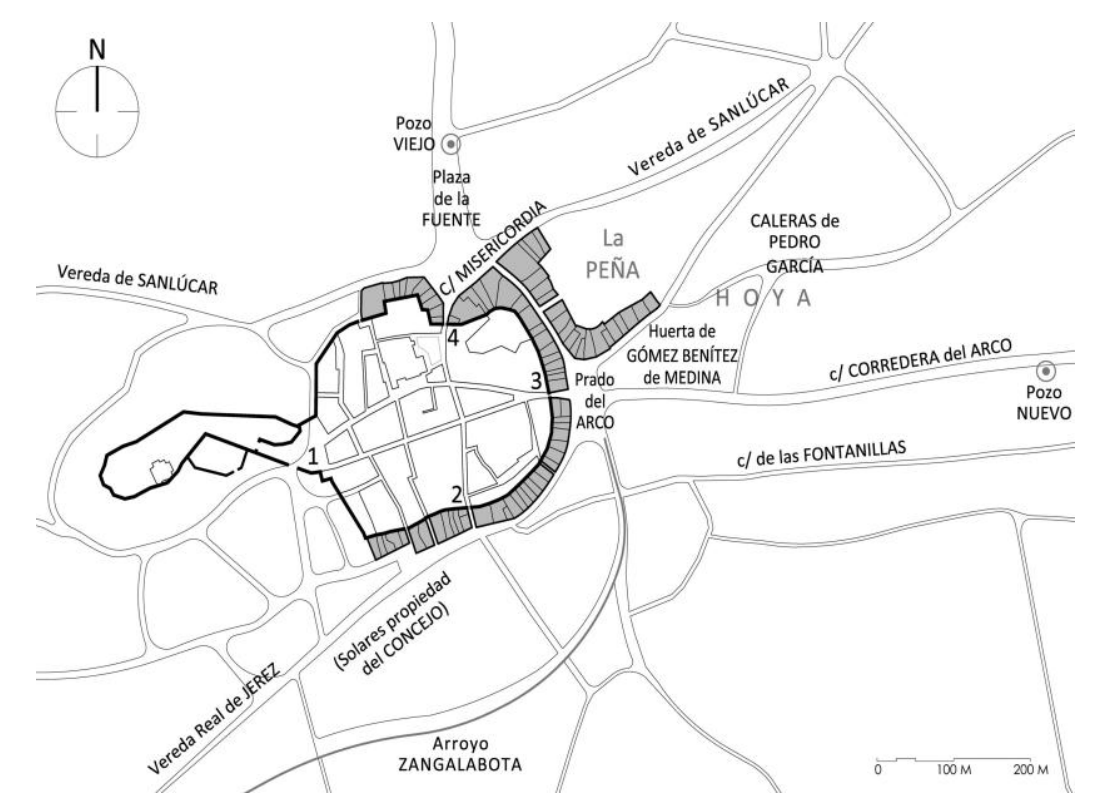

Viviendas adosadas a la muralla y primeras ocupaciones extramuros en calle Cataño y aledaños. 1: Puerta de Jerez. 2: Puerta del Aceituno o de Rota. 3: Puerta de Sevilla. 4: Puerta de Santiago.

En resumen, las direcciones matrices que formalizarían la expansión de Lebrija en el siglo XVI ya habían sido establecidas en la centuria anterior. La villa, sin embargo, está a punto de ejecutar dos operaciones urbanísticas de crecimiento independiente a los ejes antes mencionados; una, de escala reducida, se ubicará en el ámbito septentrional de la antigua cerca: el arrabal de Cantarranas. La otra, al sur de la misma, ocupará sola- 
res concejiles y muladares para conformar una amplia zona edificada cuyo topónimo es igual de significativo que el anterior: el arrabal de Barrionuevo.

\section{SIGLO XVI.}

El crecimiento urbano de Lebrija en este siglo tiene como base un aumento continuo de su población. Si para el año 1534 el censo fiscal del Reino de Sevilla arroja la cifra de 800 vecinos en Lebrija - unos 3.600 habitantes (Fernández, M. F., 2009, 64)-, encontramos que para 1596 la villa acoge una población de 1.250 vecinos, 5.626 habitantes. El último recuento ya manifiesta las consecuencias de la crisis poblacional sufrida en Castilla entre la última década del siglo XVI y principios del siglo XVII. El cénit demográfico se alcanzaría justo antes, en la década de los 70 y primeros años de los 80 del siglo XVI, con una cima de 5.749 habitantes (1.274 vecinos) para los años 1587-89 (Fernández, M. F., 2009, 69).

Una de las áreas de expansión asociado a esta cronología es el arrabal de Cantarranas. Articulado en torno a la colada de paso de la vereda de Sanlúcar, hoy calle Meneses y Portales, fue conocida antaño con el mismo nombre que el arrabal (Bellido, J., 1985, 456). Respecto a esta denominación, "Cantarranas», cabe exponer que se trata de un topónimo presente en muchas otras poblaciones, asociado siempre a zonas insalubres, lagunas, muladares, cloacas... humedales donde proliferan insectos transmisores de infecciones palúdicas y poblaciones de anfibios que originan gran ruido en horario nocturno, sobre todo en verano, época de apareamiento. En el caso de Lebrija, el topónimo está relacionado con la proximidad de las marismas. La colada de paso, o calle Cantarranas, conducía en dirección oeste hacia los cercanos terrenos palustres.

La excavación arqueológica del inmueble número 30 de Meneses y Portales arrojó una cronología de primera ocupación fechada en torno a los siglos XVI y XVII, con una construcción relacionada con actividades agropecuarias (Galván, L., 2005). En cuanto a la tipología de parcela y al modelo de implantación, asistimos a una agregación longitudinal de parcelas con lindes perpendiculares al recorrido matriz. Son lotes con fachada y fondo variables, pero que pueden agruparse en fases de tipología homogénea, y que quizás evidencian una cierta planificación secuencial a pequeña escala, sencillas operaciones de loteo que reparten el solar a edificar entre pequeños propietarios en cada ocasión, lectura que se desprende del análisis del parcelario actual.

Parte del arrabal de Cantarranas se alineaba con la explanada de la actual plaza Juan Díaz de Solís, entonces Ilamada "Campo del Príncipe» (Bellido, J., 1985, 452), y de la cual formaban parte las plazas de Manuel Halcón y Manuela Murube. Tenemos noticias desde 1480 de la existencia en esta explanada suburbana de una fuente y una pila para abrevar ganado, razón por la cual a veces era denominada «plaza de la Fuente»o «plaza del Pilar» (Bellido, J., 1985, 452).

La puerta de Santiago -el más septentrional de los accesos a la medina si excluimos el hipotético postigo de la Cuesta del Guineo-, se ubicaba en la embocadura de la calle José Sánchez de Alva. El topónimo de la puerta es compartido por otras muchas

ISSN: 0212-8594 ISSN-e: 2340-2776 № DOI: http://dx.doi.org/10.12795/rea.2011.i28.06

REA 28 (2011):93-107

http://www.publius.us.es/estudios_andaluces 
poblaciones; encontramos puertas o postigos de Santiago en Segovia, Lugo, León, Trujillo, Plasencia, o en poblaciones más cercanas como Tarifa o Jerez de la Frontera. Este

Figura 6. Restitución gráfica aproximada de Lebrija a finales del siglo XVI.

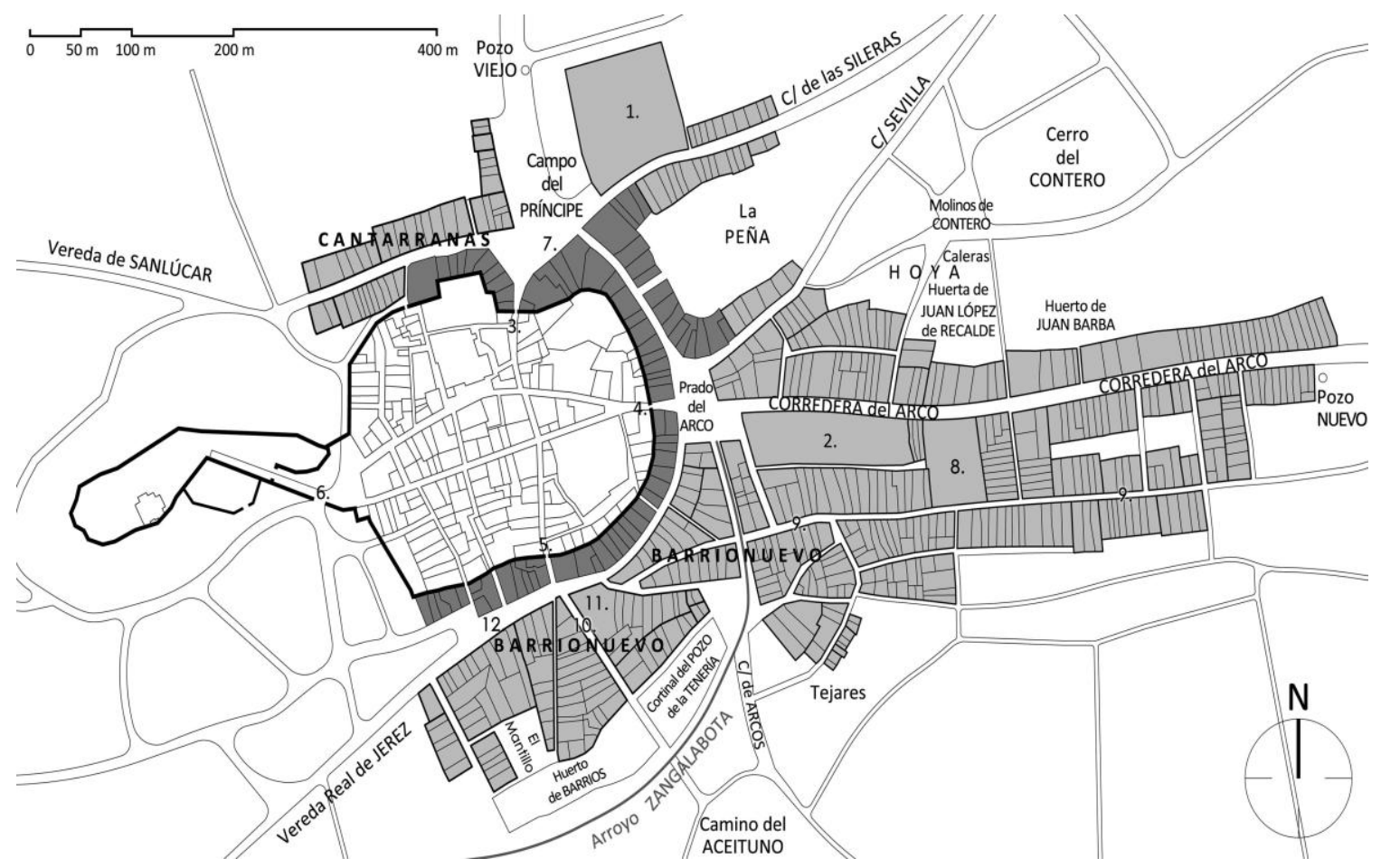

1: Convento de San Francisco. 2: Convento de Sta. María de Jesús. 3: Puerta de Santiago. 4: Puerta de Sevilla. 5: Puerta del Aceituno. 6: Puerta de Jerez. 7: Calle Juan Gil de Jarana. 8: Cortinal. 9: Calle Fontanillas. 10: Calle de los Vidales, también llamada «calle de la Barra». 11: Solar del futuro Hospital de la Caridad. 12: Actual calle Tetuán, entonces denominada de Barrionuevo.

acceso conectaba con el camino hacia Sevilla a través de las calles José Sánchez de Alva y San Francisco. La primera vía ya estaba urbanizada en el siglo XV, al menos su margen derecha; sabemos que en esta época, y desde la construcción del Hospital de la Santa Misericordia en 1496, la calle recogía el nombre de "calle de la Misericordia», y que en el siglo siguiente llevó por un tiempo el de un vecino, Juan Gil de Jarana (Bellido, J., 1985, 451). Respecto a la calle San Francisco - denominada «calle de las Sileras» quizás ya en el siglo XVI (Bellido, J., 1985, 462)—, la fundación del convento masculino homónimo hacia la década de los 70 de este siglo debió poner en valor la vía como lugar susceptible de ocupación; desconocemos, sin embargo, el alcance de los márgenes edificados, aunque no debió sobrepasar las inmediaciones del actual callejón de Borrego a juzgar por el fondo de parcela decreciente que se detecta en las medianeras y otras líneas catastrales de la acera de los pares.

La trasera de este margen, que vierte a La Peña, quedó sin edificar, como zona de huertas privadas, corrales, lugares de almacenaje o vertederos domésticos. La propia morfología rectilínea de la calle Peña sugiere su origen planificado, cuyo objeto habría sido colmatar y ordenar el vacío interior de la gran manzana delimitada por las calles 
San Francisco, Sevilla y Cataño. La Peña debió abrirse después del Quinientos, hecho que confirman las actuaciones arqueológicas llevadas a cabo en los números 21, 23 y 32 de la vía, que ofrecen una fecha hacia mediados del siglo XVII para su primera edificación (Linares, A., 2012) (figura 7).

Al sur de La Peña se encontraba en el siglo XV la huerta de Gómez Benítez de Medina, ya referida, encajada entre las calles Sevilla, Corredera, Molinos y Eduardo Dato. Ésta última era llamada «calle de la Huerta» en el siglo XVI por ser acceso a la del Contador

Figura 7. Siglo XVI.

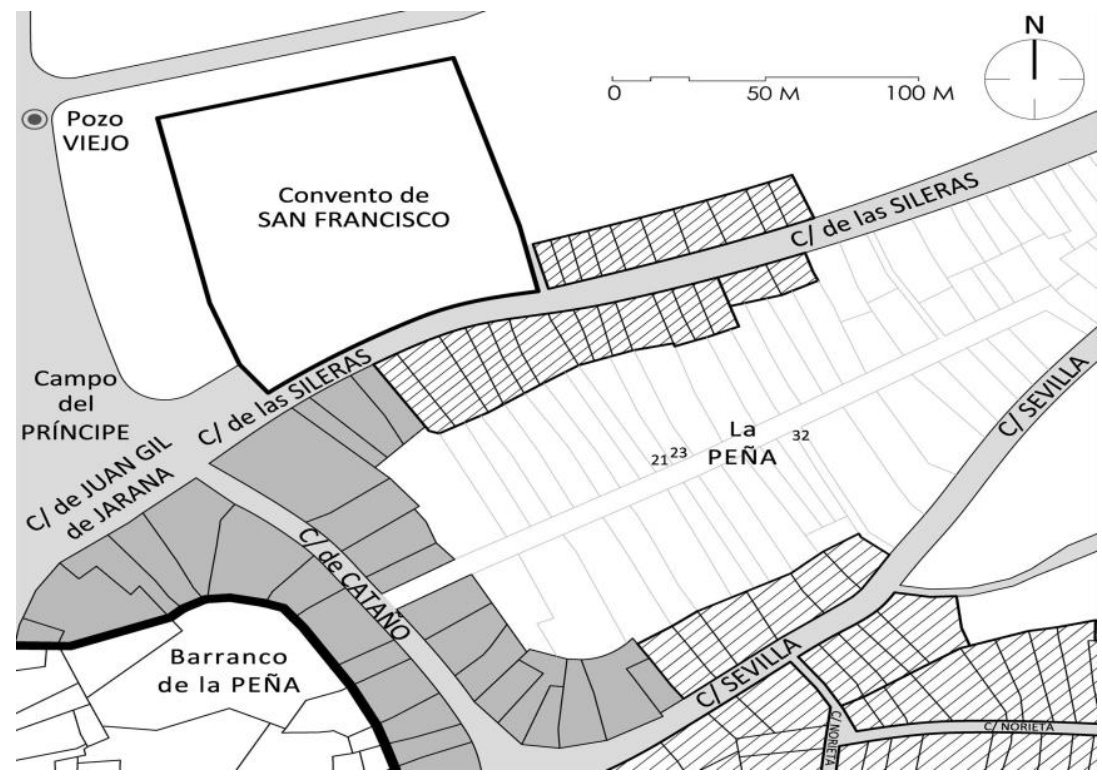

Ocupación de las calles San Francisco y Sevilla (parcelario aproximado). En el centro de la manzana se representan las parcelas de La Peña, aún sin edificar.

Mayor de la Casa de la Contratación Juan López de Recalde (Bellido, J., 1985, 447); debía tratarse por entonces de una linde rústica, una partición entre predios limítrofes con el terreno de Gómez Benítez. En todo caso, la toponimia de la época evidencia el carácter de espacio abierto del triángulo formado entre los ejes de comunicación de las calles Sevilla y Corredera. El vértice de este triángulo, que apuntaba hacia la explanada de la plaza de España - llamada «prado del Arco» o «plaza del Arco» en los siglos XV y XVI respectivamente (Bellido, J., 1985, 447) - sería la primera zona en edificarse. Así, la huerta de Gómez Benítez acabó repartida y loteada, con dos calles transversales en su interior denominadas «de la Norieta» (la actual Laudes también), en referencia a las instalaciones de riego existentes en el terreno (Bellido, J., 1985, 434).

El resto de este gran área libre entre las vías Sevilla y Corredera contiene una lectura similar a la de su vértice. El interior permaneció exento de edificaciones durante el siglo $\mathrm{XVI}^{2}$, ocupado por muladares, hornos de $\mathrm{cal}^{3}$ y molinos cuyas referencias nos lle-

\footnotetext{
${ }^{2}$ De hecho, hasta la segunda mitad del siglo XX no hallamos edificados solares como el de la calle Molinos, no 11 (Galván, L., 20120b).
} 
gan a través del antiguo y moderno callejero. Bellido refiere que El Pajarete era conocido en el Quinientos con el nombre de "Molinos de Contero», al igual que el cerro próximo. Con el tiempo, la plaza del Pajarete formalizó este vacío, apreciable aún en la planimetría catastral.

El borde perimetral, en cambio, fue objeto de ocupación, pero su alcance es impreciso. Algunas de las vías transversales, perpendiculares a la calle Sevilla, ya son nombradas en esta época. La calle Don Alonso el Sabio era conocida como la «calle de Juan

Figura 8. Siglo XVI.

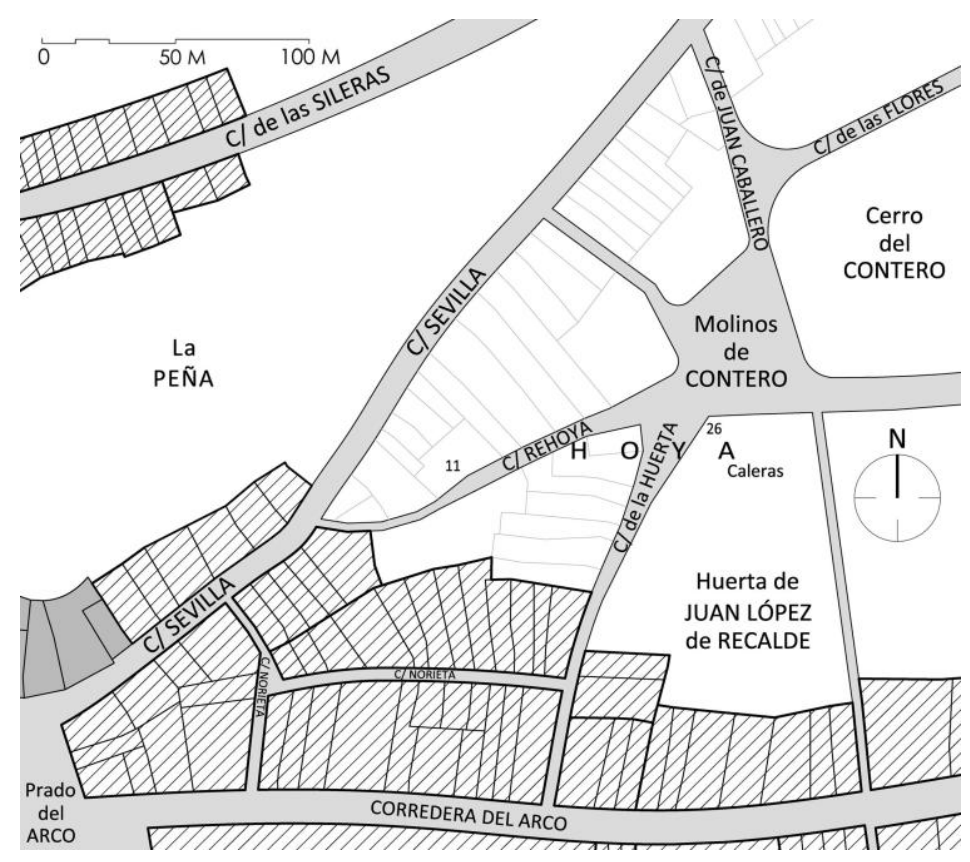

Ocupación de la antigua huerta de Gómez Benítez de Medina (parcelario aproximado). El entorno del Pajarete (entonces Molinos de Contero) sería edificado durante el siglo XVII, aunque no se descarta una ocupación incipiente; se representa su posible loteo.

Caballero» (Bellido, J., 1985, 447), y la calle Molinos compartía su título con el de "Rehoya», en referencia a una hoya, rehoyo u hondonada - desaparecida y edificada hacia los años setenta del siglo XX- que dejaba a su derecha (Bellido, J., 1985, 457) ${ }^{4}$. La calle Flores también es mencionada en este siglo (Bellido, J., 1985, 450), todo lo cual indica que la expansión urbana de la villa debía haber alcanzado esta zona, al menos de forma incipiente, lo bastante para nominar calles que sólo algunos años antes eran caminos rurales (figura 8 ).

\footnotetext{
${ }^{3}$ Aparte del topónimo medieval "Caleras de Pedro García» (Bellido, J., 1985, 459), ya comentado, se documentó un horno de cal para el siglo XVI en la excavación arqueológica preventiva llevada a cabo en la calle Eduardo Dato, no 26, esquina calle Molinos, no 24 (España, F. J., 2010).

${ }^{4}$ Bellido subraya la gran antigüedad de estos dos nombres - Molinos y Rehoya- pero no los fecha. El último no figura en ningún nomenclátor más que en el popular, y es pronunciado «rejoya».
}

ISSN: 0212-8594 ISSN-e: 2340-2776 № DOI: http://dx.doi.org/10.12795/rea.2011.i28.06

REA 28 (2011):93-107

http://www.publius.us.es/estudios_andaluces 
En cuanto a la calle Corredera, la fundación en 1510 del convento masculino de Santa María de Jesús - extramuros, al igual que el de San Francisco- ayudaría no sólo a convertir esta vía en preferente para el asentamiento edilicio sino a desplazar el foco económico y social de Lebrija desde la plaza Rector Merina a la actual plaza de España (Galván, L., 2005, 2.885). Es en la calle Corredera -o Corredera del Arco- y en su paralela Fontanillas (calle Andrés Sánchez de Alva) donde asistimos al mayor despliegue urbanístico de la centuria: 600 metros de ocupación lineal en dirección este.

No será, sin embargo, un desarrollo de tipo residencial; tenemos constancia de que estos solares serán destinados a acoger tierras baldías, huertas y cortinales (Linares, A., 2012), como indica el propio callejero del siglo XVII, donde la plaza Obispo Navarro, transversal a La Corredera, es nombrada como "calle del Cortinal» (Bellido, J., 1985, 458) en referencia al que allí tuvo Francisco de la Cerda. Así, Corredera y Andrés Sánchez de Alva serán por un tiempo calles de largas tapias con sólo unas pocas fachadas de casas intercaladas asomando a la vía pública. Durante los siglos XVII y XVIII, estas propiedades serán vendidas y segregadas en parcelas rectangulares de fondo largo y fachada estrecha con dimensiones de loteo homogéneas, resultando viviendas unifamiliares entre medianeras de superficie similar, en algunos casos con dos fachadas - una más noble hacia la vía principal y otra secundaria (como la calle Frailes)-, viviendas organizadas en torno a un patio y con una zona trasera para corral, granero o establo (Quirós, C., 2008c, 29).

Una serie de calles transversales a los recorridos principales comunican ya en el siglo XVI las calles Corredera y Andrés Sánchez de Alva, completando la trama viaria de esta zona. Su existencia está refrendada por la toponimia de la época: Chamorro, Alonso López, Almenas y Victoria se documentan (Bellido, J., 1985, 446, 433, 467). Otras vías, como el callejón del Huerto Vicario, que partía de La Corredera y daba acceso al huerto del vicario Juan Barba, o la callejuela que conducía al Pajarete rodeando las propiedades de los López de Recalde, fueron cerradas en el mismo siglo (Bellido, J., 1985, 445). Respecto a calle Nueva y a la calle Archite, debieron formarse también en el siglo XVI, si bien con un grado de urbanización parcial o nulo (Bellido, J., 1985, 439, 458).

El nuevo límite de la ciudad hacia el este quedó definido por el pozo que el Concejo de la villa mandó abrir el año 1480 (Bellido, J., 1985, 460), y que todavía da nombre a la plaza de Pozo Nuevo, anexa a Corredera. Sabemos por otro lado que la calle paralela a ésta, entonces Fontanillas, no debía ir más allá de la altura de la calle Victoria, pues la excavación en Andrés Sánchez de Alva no 124 - 126 constató que la ocupación del solar no se produjo hasta mediados del siglo XX (García, A., 2010). Pudiera ser que la acera de los pares de esta misma calle no rebasara siquiera la ubicación de la Capilla de Belén, construida entre finales del siglo XVII y principios del XVIII.

\section{BARRIONUEVO Y EL ARROYO ZANGALABOTA SIGLO XVI.}

El arco sureste de Lebrija había sido hasta finales del siglo XV una amplia zona de solares - la mayoría propiedad del Concejo de la villa (Bellido, J., 1985, 465)- destinados a huertas y a vertederos, y encajados entre el camino de la vereda hacia Jerez, actual

ISSN: 0212-8594 ISSN-e: 2340-2776 № DOI: http://dx.doi.org/10.12795/rea.2011.i28.06

REA 28 (2011):93-107

http://www.publius.us.es/estudios_andaluces 
calle Tetuán, y el arroyo Zangalabota, que discurría de manera aproximada por el primer tramo de la actual calle Arcos para girar en dirección oeste por la calle Arroyo Zangalabota. Este uso preexistente fue sustituido por edificaciones de tipologías residencial y productiva, y algunos edificios singulares, tanto civiles como religiosos. El joven arrabal se denominó «Barrionuevo».

La primera fase de su construcción debió crearse de manera espontánea en torno a la actual plaza de España, para luego descender por la vereda de Jerez. No obstante, la calle Tetuán y su continuación calle Trinidad quedaron nombradas, por tramos y en sentido inverso, como "Barrionuevo Primero», "Barrionuevo Segundo» y "Barrionuevo Tercero». No todos los solares fueron edificados en el Quinientos; el número 31 de la calle Tetuán conservó su uso marginal como basurero doméstico hasta la construcción del Hospital de la Caridad a finales del siglo XVII (Pérez, M., 2005). Junto a esta finca se abrirían dos calles cuya geometría rectilínea lleva a pensar - como en el caso de la calle Peña - en un trazado a cordel: son las calles Luis Collado, cuyo nombre en el siglo XVI era "calle de los Vidales», que alternaba con «calle de la Barra» (Bellido, J., 1985,

Figura 9. Siglo XVI.

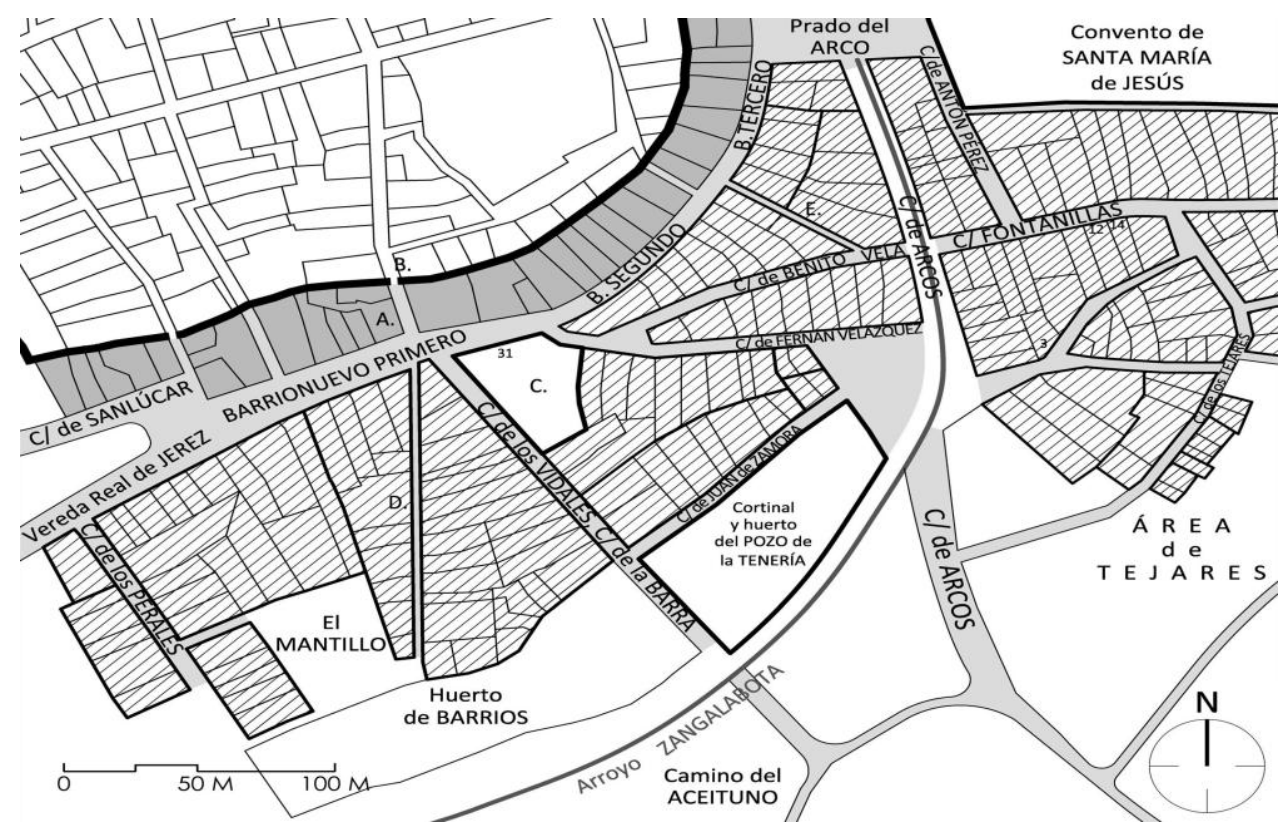

Arrabal de Barrionuevo (parcelario aproximado). A: Capilla de la Veracruz. B: Puerta del Aceituno o de Rota. C: Hospital de la Caridad (construido a finales del siglo XVII). D: Callejuela de Marín. E: Calleja de García Martín Romo.

454, 455), y la calle de los Marines, llamada entonces «callejuela de Marín» (Bellido, J., $1985,456)$. El origen planificado es justificable, al menos en el primer caso -el carácter ex novo de la formación del arrabal daba ocasión a ello-, aunque no se descarta que la calle Luis Collado consolidara y formalizara un paso ya existente desde el camino del Aceituno hasta la puerta del mismo nombre, llamada también «puerta de Rota», situada aproximadamente a la entrada de la calle Cala de Vargas. También la calle Perales —así llamada ya a principios del siglo XVI (Bellido, J., 1985, 460) - goza de la 
pureza formal del viario proyectado de nueva planta. Esta última calle vendría a ser el límite occidental aproximado del arrabal. La linde sur - borde de contacto con el arroyo Zangalabota-, conservó durante toda la centuria, y aun después, algunos solares destinados al cultivo periurbano, como el «huerto de Barrios» y el "cortinal del Pozo de la Tenería» (Bellido, J., 1985, 438), y espacios libres residuales como la actual plaza del Mantillo (figura 9).

Dos puentes cruzaban la parte alta del riacho de Zangalabota. Era un arroyo de cauce profundo (Bellido, J., 1985, 439), fisonomía en la que es posible radique su nombre si aceptamos un hipotético «zanja» como hidrónimo de partida.

Las calles transversales de Barrionuevo que asoman a este curso ya están recogidas en el siglo XVI. Fernán Velázquez y Benito Vela conservan aún sus nombres originales (BeIlido, J., 1985, 449, 440), al igual que la adyacente de Antón Pérez, conocida desde el último cuarto de la centuria (Bellido, J., 1985, 436).

Mientras la parte de Barrionuevo más cercana a la muralla era ocupada con viviendas de propietarios medianos, edificios singulares y de abastecimiento, la margen oriental del río Zangalabota fue ganada para inmuebles de uso industrial y casas de pequeños propietarios, si bien en una fecha posterior al Quinientos, como apuntan la datación de la almazara excavada en la calle Andrés Sánchez de Alva número 14 y la vigilancia hecha en el solar anexo, número 12 (España, F. J., 2009). El propio viario de esta zona de crecimiento, adscribible aún a Barrionuevo, presenta un nomenclátor tardío documentado no antes en el siglo XVII, según Bellido, lo cual manifestaría un tejido urbano más débil que dilató su afianzamiento. El límite de este sector, eso sí, parece marcarlo la calle Tejar, así nombrada - «calle de los Tejares», y también "calle de las Cantarerías»- desde el siglo XVI (Bellido, J., 1985, 465). Junto a ella se levantaron talleres cerámicos, hornos y testares de piezas de barro cocido.

\section{RESTITUCIÓN GRÁFICA.}

La principal dificultad en el estudio del urbanismo antiguo de cualquier población radica en la simple inexistencia de planimetría. Las primeras representaciones cartográficas sistematizadas de ciudades europeas surgen en el siglo XV a la luz del Renacimiento, pero llegan a la Península casi dos siglos más tarde.

Se ha planteado en este trabajo, a modo de herramienta para el estudio, una restitución planimétrica no sólo de la estructura urbana y la red viaria de Lebrija en el siglo $\mathrm{XVI}$, sino incluso de su parcelario aproximado, rastreándose para ello las líneas catastrales primitivas - traseras, lindes, medianeras de loteo, etc.-, muchas de ellas aún presentes y fosilizadas en la topografía urbana actual.

El resultado es una instantánea hipotética de la ocupación extramuros de la villa a finales del siglo XVI, visión de conjunto que atiende igualmente a aspectos formales del 
espacio contenido por la cerca urbana ${ }^{5}$. Todo lo cual ayudará a comprender e imaginar la villa en este momento de su historia: un sencillo caserío - en su mayor parte de escasa altura y aún con una fuerte impronta rural- extendiéndose a los pies del antiguo cinturón defensivo fagocitado ahora por viviendas, con una estructura de implantación libre de hipotecas medievales, pero condicionada por la red de flujos del territorio, la orografía y la red hidrológica del terreno de asentamiento.

\section{BIBLIOGRAFÍA.}

Amaya hidalgo, R. L., et álii (2010): "Intervención arqueológica preventiva en la calle Porvera, no 5 (Jerez de la Frontera, Cádiz)". Anuario arqueológico de Andalucía 2006, pp. 412 - 418. Consejería de Cultura, Junta de Andalucía.

Bellido Ahumada, J. (1985): "La patria de Nebrija. Noticia histórica". Los Palacios, Sevilla.

España Caparrós, F. J., et álii (2009): "Vigilancia arqueológica de movimientos de tierra en calle Andrés Sánchez de Alva, no 14. Lebrija (Sevilla)". Anuario arqueológico de Andalucía 2004, Vol. 1, pp. 3.314 - 3.319. Consejería de Cultura, Junta de Andalucía.

España Caparrós, F. J., et álii (2010): “Actuación arqueológica preventiva. Control arqueológico de movimiento de tierras en calle Eduardo Dato, $n$ - 26, esquina calle Molinos, no 24. Lebrija (Sevilla)". Anuario arqueológico de Andalucía 2006, pp. 3.918 3.926. Consejería de Cultura, Junta de Andalucía.

Fernández Chaves, M. F. (2009): "La Villa de Lebrija a finales del reinado de Felipe III. EI padrón de Bula de Santa Cruzada de 1620", Revista de Humanidades, no 16, pp. 59 90.

García Mancha, A., et álii (2010). "Actuación arqueológica preventiva. Control arqueológico de movimientos de tierra en calle Andrés Sánchez de Alva, no 124 - 126. Lebrija (Sevilla)". Anuario arqueológico de Andalucía 2006, pp. 3.939 - 3.948. Consejería de Cultura, Junta de Andalucía.

Galván Montes, L., et álii (2009): "Intervención arqueológica preventiva. Vigilancia arqueológica de movimientos de tierras. c/ Tetuán no 41. Lebrija (Sevilla)". Anuario arqueológico de Andalucía 2004, Vol. 1, pp. 3.320 - 3.325. Consejería de Cultura, Junta de Andalucía.

\footnotetext{
${ }^{5}$ Sirva de ejemplo la representación del acceso a la puerta de Sevilla en la calle Ignacio Halcón. Este viario fue objeto de realineación en 1868, operación que eliminó el acceso en forma de embudo de la calle, similar al que hoy día se dispone en la calle Sánchez de Alva, y que da acceso a la puerta de Santiago.
} 
Galván Montes, L., et álii (2010a): "Actividad arqueológica preventiva. Control arqueológico de movimientos de tierra en calle Meneses y Portales, no 30. Lebrija (Sevilla)". Anuario arqueológico de Andalucía 2005, pp. 2.883 - 2.888.

Galván Montes, L., et álii (2010b): "Actuación arqueológica preventiva. Control arqueológico de movimientos de tierra en calle Molinos, no 11. Lebrija (Sevilla)". Anuario arqueológico de Andalucía 2005, pp. 2.889 - 2.893. Consejería de Cultura, Junta de Andalucía.

Linares Agüera, A. (2012): "Plan General de Ordenación Urbanística de Lebrija (SeviIla)". Documento para aprobación inicial (noviembre de 2012). Consejería de Agricultura, Pesca y Medio Ambiente, Junta de Andalucía.

Pérez Sánchez, M., et álii (2005): “Control arqueológico y análisis paramental realizado en el Hospital de la Caridad de Lebrija (Sevilla)". Anuario arqueológico de Andalucía 2002, pp. 499 - 507. Consejería de Cultura, Junta de Andalucía.

Del Pozo Y Barajas, A. (1996): “Arrabales de Sevilla, morfogénesis y transformación. El arrabal de los Humeros", Universidad de Sevilla, Fundación Fondo de Cultura de SeviIla, Consejería de Obras Públicas y Transportes, Junta de Andalucía.

Quirós Esteban, C. A., et álii (2001): “Excavación arqueológica del Castillo (Lebrija, SeviIla)". Anuario arqueológico de Andalucía 1998, pp. 1.022 - 1.031. Consejería de Cultura, Junta de Andalucía.

Quirós Esteban, C. A. (2008a): "Lebrija. Apuntes de historia y paisaje urbano". Aparejadores, no 74, pp. 20 - 23. Sevilla, Colegio Oficial de Aparejadores y Arquitectos Técnicos de Sevilla.

Quirós Esteban, C. A. (2008b): "Paisajes culturales de Lebrija". Aparejadores, no 74, pp. 38 - 39. Sevilla, Colegio Oficial de Aparejadores y Arquitectos Técnicos de Sevilla.

Quirós Esteban, C. A., et álii (2008c): "El avance del Plan Especial del Conjunto Histórico de Lebrija (Sevilla). Oportunidades y retos de la ciudad histórica". Aparejadores, no 74, pp. 24 - 33. Sevilla, Colegio Oficial de Aparejadores y Arquitectos Técnicos de Sevilla.

Tomassetti Guerra, J. Mạ. (1996): “Contribución de estudio urbanístico de la Lebrija antigua y medieval: los hallazgos del barrio de Cantarranas". Estudios de Historia y de Arqueología medievales, № 11, pp. 453 - 480.

Tomassetti Guerra, J. Ma. (1997): "Contribución al estudio del urbanismo antiguo en el Bajo Guadalquivir: el caso de Lebrija (Sevilla)". SPAL: Revista de Prehistoria y Arqueología de la Universidad de Sevilla, № 6, pp. 243-262. 